\title{
Moisture diffusion coefficient estimation in peas drying by means of a modified Hawlader and Uddin method
}

\section{Martínez-Vera, C.*; Vizcarra-Mendoza, M. G.}

Departamento de Ingeniería de Procesos e Hidráulica, Universidad Autónoma MetropolitanaIztapalapa. Apartado Postal 55-534, México D.F., 09340, México.

*E-mail of the corresponding author: $\underline{\text { cmv@xanum.uam.mx }}$

\begin{abstract}
The aim of the present work is to determine the moisture diffusion coefficient in peas applying, in a first step, a methodology previously published in the literature by Uddin et al. ${ }^{[1]}$ for determining constant diffusion coefficients taking in account the volume reduction associated to the drying process. Then, in a second step, refine it by means of an optimization step. The optimization step is justified because the methodology of Uddin et al. is based in a solution of the diffusion equation that is not mathematically valid for the drying-shrinking problem.
\end{abstract}

Keywords: : moisture diffusivity; drying-shrinking; peas drying. 


\section{Introduction}

Usually bio-products such as fruits and vegetables have high initial moisture content and present appreciable volume shrinkage during the drying process. Shrinkage of biological materials takes place simultaneously with moisture diffusion during drying and thus may affect the drying rate. It is important to take into account the shrinking phenomena when determining the moisture diffusion coefficients. When this phenomenon is not negligible there are not analytical solutions available for the equations describing this moisture diffusion process. In these cases changes in radius should be taken into consideration when predicting moisture profiles and, also when estimating diffusion coefficients. Not taking into account volume shrinkage leads to over-estimated diffusion coefficients (Xianxi et al. ${ }^{[2]}$; Guohong et al. ${ }^{[3]}$ ).

A simple method for determining diffusion coefficients taking into account the variation in radius was proposed by Uddin et al. ${ }^{[1]}$. It is based on a modification to the one term truncated infinity series solution that takes into account the reduction in radius. Examples of its application to drying can be found in Hawlader et al. ${ }^{[4]}$, Giovanelli et al. ${ }^{[5]}$, García et al. ${ }^{[6]}$, Martínez et al. ${ }^{[7,8]}$.

The aim of the present work is to determine the moisture diffusion coefficient in peas applying, in a first step, the methodology previously published in the literature by Uddin et al. $^{[1]}$ for determining constant diffusion coefficients taking into account the volume reduction associated to the drying process. Then, in a second step, the diffusivity values are adjusted in order to satisfy a more rigorous model of the drying-shrinking problema by means of an optimization procedure. The optimization step is justified because the methodology of Uddin et al. is based on a solution of the diffusion equation that is not valid for drying-shrinking problems. Finally, in a third step, by means of another optimization step, the model of the shrinking phenomena is improved.

\section{Materials and Methods}

\subsection{Uddin methodology}

Mass transfer processes are commonly described by the second Fick's law, the phenomenological parameter in this equation is the mass diffusion coefficient. Analytical solutions to the diffusion equation for mass transfer in solids are available for some cases under certain assumptions among which could be mentioned constant volume and constant diffusivity. In particular, for unidirectional mass transfer in slabs or spheres with a symmetry boundary condition at the centre and a Dirichlet boundary condition at the external border, with uniform initial diffusant content, these solutions are given in the form of infinite Fourier series and are the departing point for the determination of constant moisture diffusion coefficients at a constant temperature from the truncated to just one term or at most to a few terms of the series when volume shrinkage is not relevant. A method presented in the literature for determining diffusion coefficients taking into account the radius variation was proposed by Uddin et al. ${ }^{[1]}$. It is based on a modification of the one term truncated infinity series solution to take into account the reduction in radius. 
In the Uddin et al. methodology the moving boundary condition is introduced a posteriori in the solution for the fixed boundary condition. As signalled properly by Hawlader et al. ${ }^{[4]}$ this approach is a simplified one and the diffusivity coefficient determined by his method can be considered an effective diffusivity. However, the methodology provides an effective diffusion coefficient of the specimen under given drying conditions which is valid for the assumed model.

The Uddin et al. approach departs from an approximated solution to the moisture transfer in the solid when the shrinkage is neglected. The analogous equations for spherical coordinates analogus to those presented by Uddin et al. for a slab are :

$$
\operatorname{Ln}\left(\frac{c}{c_{0}}\right)=\operatorname{Ln}\left(\frac{6}{\pi^{2}}\right)-\frac{\pi^{2} D t}{R^{2}}
$$

Where $c$ indicates de volumetric moisture concentration $\left(\mathrm{kg} / \mathrm{m}^{3}\right), c_{e q}$ the equilibrium moisture content, $c_{0}$ the initial moisture content, $R$ the particle radius $(\mathrm{m})$ Then $D$, the moisture diffusion coefficient, could be obtained from the slope of a plot of $\operatorname{Ln}\left(\omega / \omega_{0}\right)$ versus $t$ or $t / R^{2}$ for those cases in which the shrinkage is negligible. For those cases for which this condition is not sustainable the proposed methodology proceeds as follows. The reduced radius $R$ ' at time $t$ was related to the moisture content at each time instant by the following equation:

$$
\frac{R I}{R}=\left(\frac{\omega_{t}}{\omega_{0}}\right)^{n}
$$

Where $R_{0}$ is the radius at the initial time, $R^{\prime}$ is the reduced radius, $\omega_{0}$ is the initial moisture content (kg water $/ \mathrm{kg}$ dry mass) and $\omega_{t}$ is the moisture content at time $t . n$ is an exponent to be found which makes that the plot of $\operatorname{Ln}\left(\omega_{/} / \omega_{0}\right)$ versus $t / R^{\prime}{ }^{2}$ results in almost a straight line. A value of $n$ equal 0 means there is not shrinkage and a value of $n=1$ would indicate that the reduction in volume is equal to the volume of the water transferred to the air.

In the present work we start with the diffusion coefficients obtained with the Uddin et al. approach. In the second step the drying process is posed, as a direct moving boundary problem in which the diffusion coefficient is assumed as known and with experimental radius-time data. The model (given below) is numerically solved, the average moisture concentration at each integration time step is obtained and the average moisture content profile is compared with the experimental results. If the difference between the experimental and the predicted profile is beyond certain tolerance according to a predefined norm the diffusion coefficient is improved through an optimization algorithm until the desired tolerance is satisfied. The model is implemented in the software Berkeley Madonna discretizing the spatial variable of the distributed parameter model by finite differences and integrating the set of ordinary differential equation with one of the integration methods available in the software. The optimization step is carried on by means of the optimization algorithm with which Berkeley Madonna is provided with experimental radius-time data. The optimization procedure is carried on taking as objective function the minimization of the difference between the predicted moisture content and the experimental one at the time for which this difference is higher. After finding the optimal diffusion with experimental radius-time data, in the third step, by means of another optimization procedure, the model of the shrinking phenomena is improved taking as objective function the minimization of 
the difference between the predicted radius and the experimental one at the time for which this difference is higher.

\subsection{Model of the drying process considering shrinkage}

Assuming that the drying process is described by the diffusion equation, considering that the concentration is a function of volume and time, that the volume is as well a function of time and, after transforming the moving boundary problem into a fixed boundary problem by means of a change of variable $(z=r / R)$ in order to have variations of the dimensionless spatial variable between 0 and $1\left(\operatorname{Crank}^{[10]}\right)$, the following equation is obtained:

$$
\frac{d c}{d t}=\frac{1}{(z \cdot R)^{2}} \frac{\partial}{\partial z}\left(z^{2} \cdot D \frac{\partial c}{\partial z}\right)+\frac{1}{R} \frac{\partial c}{\partial z} z \frac{d R}{d t} \quad 0 \leq z<1, t>0
$$

where $r$ denotes the radial distance from the centre of the sphere $(m)$.The consideration of a spherical particle leads to a symmetry condition at the centre of it:

$$
\frac{\partial c}{\partial z}=0 \quad z=0, \forall t
$$

An equilibrium condition is assumed at the solid-gas interface:

$$
c=c_{e q} \quad z=1, \forall t
$$

A uniform moisture content profile inside the solid is assumed at the initial time:

$$
c=c_{0} \quad 0 \leq z<1, t=0
$$

Assuming that the solid's volume reduction is proportional to the volume of water lost by it (Aguerre et al. ${ }^{[11]}$ ), the following equation gives the shrinking rate

$$
\frac{d R}{d t}=-\frac{\beta D}{\rho_{w} R}\left[\frac{\partial c}{\partial z}\right]_{z=1}
$$

Where $\beta$ is a proportionality constant introduced in order to fit the model predictions with the experimental data to be found. The initial condition for this equation is:

$$
R=R_{0}, t=0
$$

The average volumetric moisture concentration $\bar{c}(t)$ is given by

$$
\bar{c}(t)=\frac{1}{V_{p}} \int_{0}^{V_{p}} c(r, t) d v
$$

In order to solve the above equations when the diffusion coefficient is assumed to be known, the spatial variable is discretized by finite differences leading to a set of ordinary differential equations which are integrated with a proper method for stiff equations. 


\section{Results and discussion}

This work departs from experimental data obtained in a small scale batch fluidized bed drier for drying of peas (Gómez et al. ${ }^{[12]}$ ). Peas were dried at three different temperature levels of the air fed to the drier: 50,60 and $70^{\circ} \mathrm{C}$. The moisture content and the diameter of the peas were recorded along the drying time. These data were fitted by analytical expressions in order to have available the moisture content and the diameter at each time instant during the drying process.

Table 1. Diffusion coefficient and adjusted exponent in eq. (2) obtained with the Uddin method.

\begin{tabular}{ccc}
\hline $\mathrm{T}\left[{ }^{\circ} \mathrm{C}\right]$ & $n$ & $D_{\text {ef }} \times 10^{8}\left[\mathrm{~m}^{2} \mathrm{~s}^{-1}\right]$ \\
\hline 40 & 0.75 & 3.04 \\
50 & 0.55 & 4.053 \\
60 & 0.55 & 3.04 \\
70 & 0.45 & 1.013
\end{tabular}

The effective moisture diffusion coefficients of peas dried at each one of the temperature levels at which the experiments were carried on were determined applying the methodology proposed by Uddin et al. described above. The values obtained are presented in Table 1 (Martínez et al. ${ }^{[8]}$ ). Posteriorly, the constant values obtained were tested in the more rigorous model of the drying process represented by equations (3a-3d and 4). These equations were solved discretizing the spatial variable (radius) by finite differences and the set of ordinary differential equations were integrated together with the boundary conditions by means of an integration method proper for stiff equations. In these simulations the experimental radius values corresponding to each integration time step were provided by the corresponding fitted expression to the radius-time experimental values. These values ofthe diffusion coefficients, as expected, led to poor predictions of the moisture evolution as shown by the dashed lines in Figs. 1, 2 and 3 which can be compared with the solid ones which represent the experimental values. The diffusion coefficients obtained are between $1 \times 10^{-8}$ and $4 \times 10^{-8} \mathrm{~m}^{2} \mathrm{~s}^{-1}$.
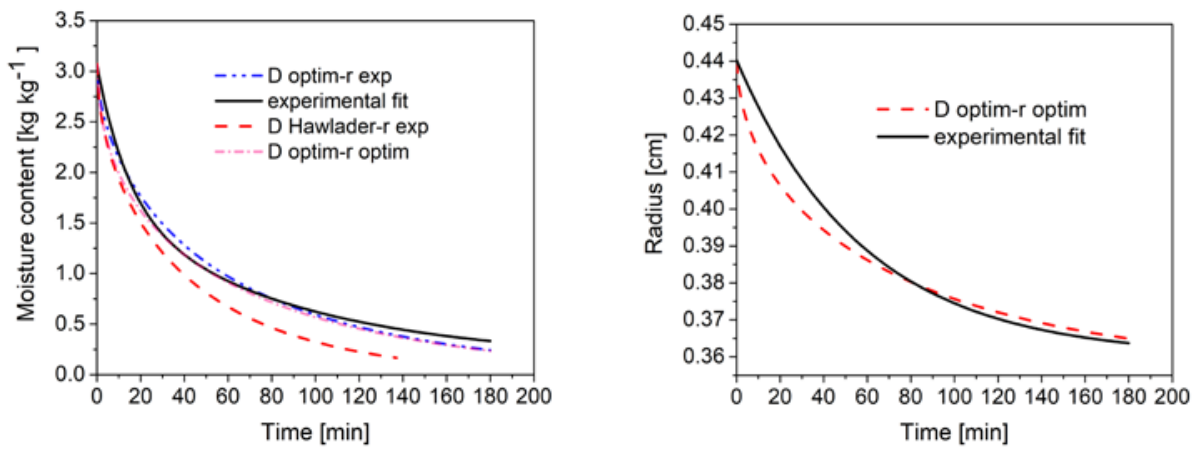

Fig. 1. Moisture content profiles and experimental and predicted radius profiles at inlet air temperature of $50^{\circ} \mathrm{C}$. 
In a second step the diffusion coefficients were improved in order to give a better agreement with the experimental moisture content profiles when the model given by equations (3a-3d and 4) was employed. This was done by an optimization procedure utilizing the optimization tool included in the software Berkeley Madonna. In this optimization step the experimental radius values given by the radius-time fitted expression were employed. The objective function to be minimized was the absolute value of the difference between the moisture content experimental value and the predicted one at each integration time step. The variable to be optimized was the effective diffusion coefficient. The profiles obtained with the optimized values in the rigorous model but still with the experimental radius values are shown in figures 1, 2 and 3 by the dash-dot-dot-dash lines. The improvement in the agreement between the experimental moisture content profile and the predicted by the simulations can easily be appreciated.
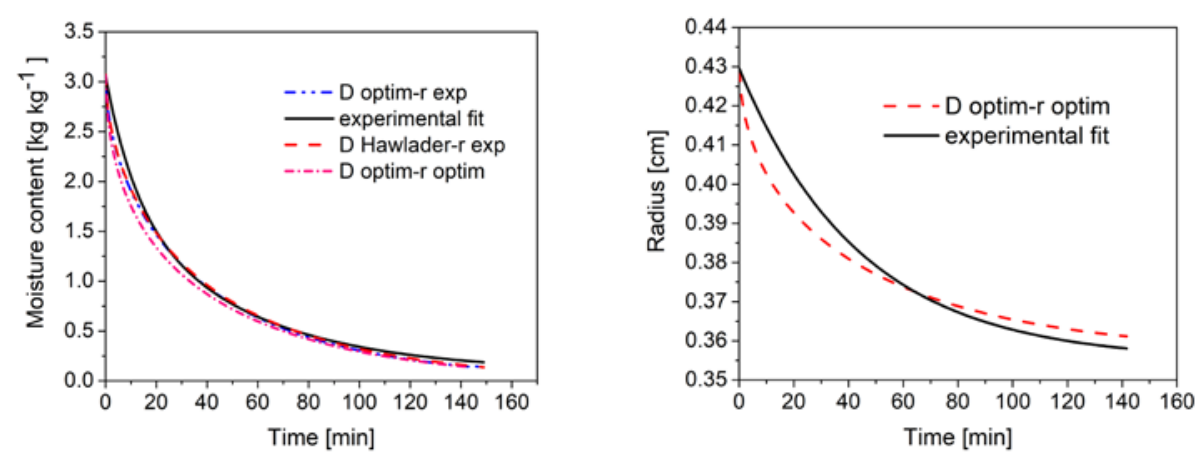

Fig. 2 Moisture content profiles and experimental and predicted radius profiles at inlet air temperature of $60^{\circ} \mathrm{C}$.

However, even though the moisture content predictions were quite acceptable, the radius evolution predictions given by equations (3e-3f) were quite poor. Therefore, a third step was carried on in order to improve the particle size predictions. This was done through a second optimization step with the optimization algorithm included in the software Berkeley Madonna in which the variable to be optimized was the parameter $\beta$ introduced in equation (3e). The objective function to be minimized was the absolut value of the difference between the experimental radius and the predicted one at the time in which this difference was bigger before the optimization. The results in the moisture content are shown in figures 1,3 and 5 by the short dash-dot-short dash lines. It can be appreciated that the predictions of the moisture content profiles employing the experimental radius values and those obtained with radius values given by the model does not differ appreciably. The radius profiles obtained with equations (3a-3f and 4) and the experimental ones are shown, for each temperature level considered in this work, in figures 1, 2 and 3. It can be seen that, even though, the particle size predictions are not as good as the moisture content predictions, they are acceptable. 

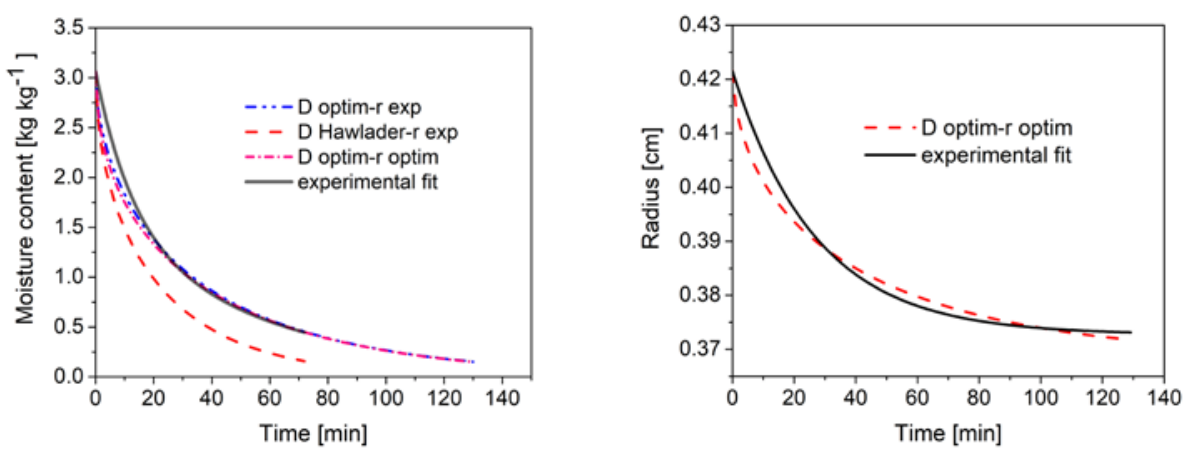

Fig. 5 Moisture content profiles and experimental and predicted radius profiles at inlet air temperature of $70^{\circ} \mathrm{C}$.

\section{Conclusions}

A methodology proposed by Udin and Hawlader for the determination of moisture diffusion coefficients taking into account the shrinkage of the stuff being dried that is easily applied provides diffusivity values that are taken as first guesses of an optimization procedure under a more rigorous model. Having obtained the optimal moisture diffusion coefficients employing the experimental radius evolution data a second optimization step is carried on in order to obtain an acceptable prediction of the radius evolution. The results obtained shows a very good agreement with the experimental moisture content evolution at the three temperature levels analysed and an acceptable agreement with the experimental radius evolution.

\section{References}

[1] Uddin M. S.; Hawlader M. N. A.; Rahman S. Evaluation of Drying Characteristics of Pineapple in the production of pineapple powder. Journal of Food Processing and Preservation 1990, 14, 375-391.

[2] Xianxi Liu; Junruo Chen; Meihong Liu; Ziliang Li; Yifei Tao; Daigen Zhu. (2010), The Effect of Biological Material's Tissue Shrinking on Moisture Diffusivity during Hot-air-drying. In Proceedings of the World Automation Congress, Kobe, Japan, 2010; 255-260.

[3] Guohong Liu; Junruo Chen; Meihong Liu; Xinxin Wan. Shrinkage, porosity and density behavior during convective drying of bio-porous material. Procedia Engineering 2012, 31, 634-640.

[4] Hawlader M. N. A.; Uddin M. S.; Ho J. C.; Teng A. B. W. Drying Characteristics of Tomatoes. Journal of Food Engineering 1991, 14, 259-268.

[5] Giovanelli G.; Zanoni B.; Lavelli V.; Nani R. Water Sorption, drying and antioxidant properties of dried tomato products. Journal of Food Engineering 2002, 52, 135-141. 
[6] García-Hernández A., M. Vizcarra-Mendoza, C. Martínez-Vera, H. Vázquez-Torres. Thin Layer Drying of Tomato Cubes in a Dryer Tunnel. In Proceedings of the Sixth Nordic Drying Conference, Copenhague, Denmark, June 5-7, 2013.

[7] Martínez Vera, C.; Anaya Sosa, I.; Vizcarra Mendoza, M. G. Diffusion Coefficient Estimation in Shrinking Solids. A Case Study: Tomato. American Journal of Food Science and Technology 2015, 5 (3), 132-136.

[8] Martínez Vera C.; Vizcarra Mendoza, M.G.; Samperio Domínguez, L. A. ; Gómez Rojas, H. M. Concentration dependent diffusion coefficient estimation in peas and cranberries drying considering shrinkage: an observer approach. In Proceedings of the Second Nordic Baltic Drying Conference. Hamburg, Germany, June 7-9, 2017.

[9] Crank, J. The Mathematics of Diffusion (2nd ed.); Oxford University Press: 1975.

[10] Aguerre R. J.; Tolaba M.; Suárez C. Modeling volume changes in food drying and hydration, Latin American Applied Research 2008, 38, 345-349.

[11] Gómez Rojas, H. M.; Arredondo Bote, G. M.; Mar Antonio, S. I. , México, 2016.Desarrollo de un proceso para producir chicharos secos para su comercialización y conservación. B.Ch.E. Thesis. Universidad Autónoma Metropolitana. 